\title{
Concepts for Autonomous Operation of Microreactors
}

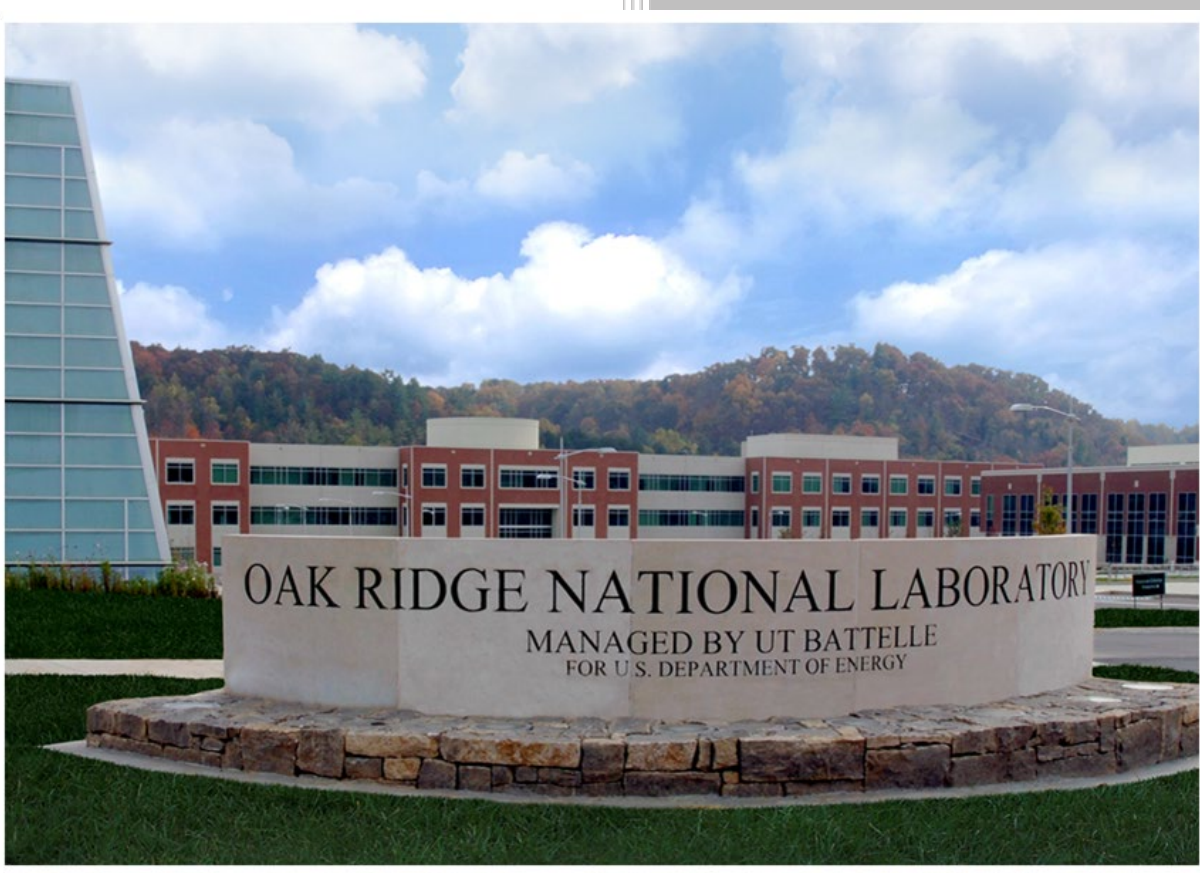

Approved for public release.

Distribution is unlimited.

P. Ramuhalli

S. M. Cetiner

September 30, 2019 


\section{DOCUMENT AVAILABILITY}

Reports produced after January 1, 1996, are generally available free via US Department of Energy (DOE) SciTech Connect.

Website www.osti.gov

Reports produced before January 1, 1996, may be purchased by members of the public from the following source:

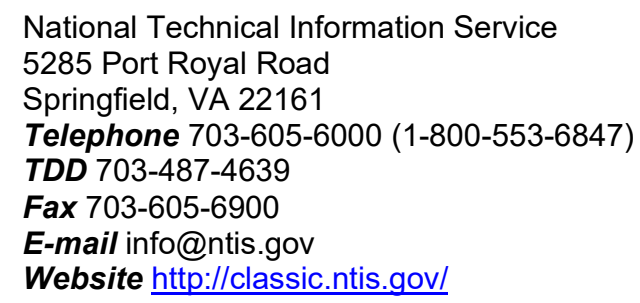

Reports are available to DOE employees, DOE contractors, Energy Technology Data Exchange representatives, and International Nuclear Information System representatives from the following source:

Office of Scientific and Technical Information

PO Box 62

Oak Ridge, TN 37831

Telephone 865-576-8401

Fax 865-576-5728

E-mail reports@osti.gov

Website http://www.osti.gov/contact.html

This report was prepared as an account of work sponsored by an agency of the United States Government. Neither the United States Government nor any agency thereof, nor any of their employees, makes any warranty, express or implied, or assumes any legal liability or responsibility for the accuracy, completeness, or usefulness of any information, apparatus, product, or process disclosed, or represents that its use would not infringe privately owned rights. Reference herein to any specific commercial product, process, or service by trade name, trademark, manufacturer, or otherwise, does not necessarily constitute or imply its endorsement, recommendation, or favoring by the United States Government or any agency thereof. The views and opinions of authors expressed herein do not necessarily state or reflect those of the United States Government or any agency thereof. 
Reactor and Nuclear Systems Division

\title{
Concepts for Autonomous Operation of Microreactors
}

\author{
P. Ramuhalli \\ S. M. Cetiner
}

Date Published: September 2019

\author{
Prepared by \\ OAK RIDGE NATIONAL LABORATORY \\ Oak Ridge, TN 37831-6283 \\ managed by \\ UT-BATTELLE, LLC \\ for the \\ US DEPARTMENT OF ENERGY \\ under contract DE-AC05-00OR22725
}





\section{CONTENTS}

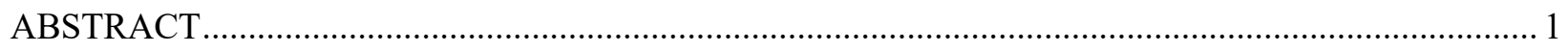

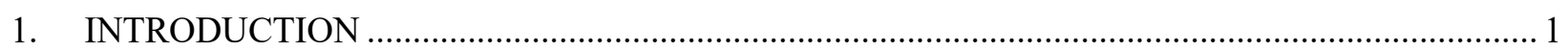

2. AUTONOMOUS OPERATION OF MICROREACTORS: REQUIREMENTS ............................... 3

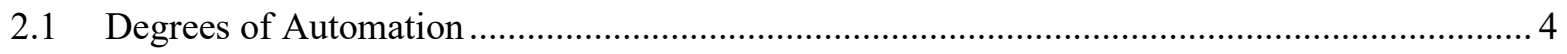

2.2 Motivation for Autonomous Operation of Microreactors ................................................... 5

2.3 Initial High-Level Requirements for Autonomous Operation ............................................... 6

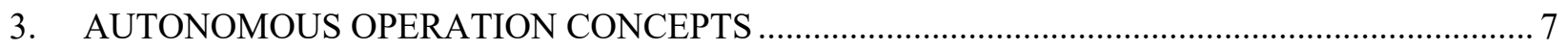

3.1 Generic Framework for Autonomous Operation .............................................................. 7

3.2 Transformational Challenge Reactor for Demonstration of

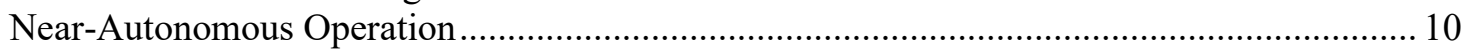

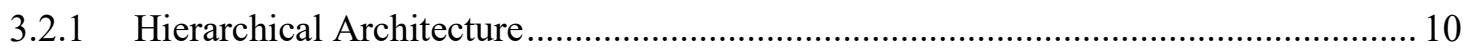

4. AUTONOMOUS OPERATION OF MICROREACTORS: POTENTIAL APPROACHES AND DESIRED TECHNICAL ADVANCES ................................................... 12

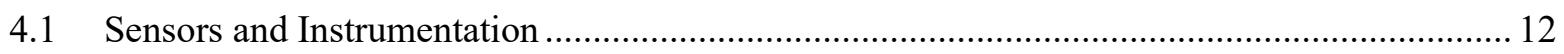

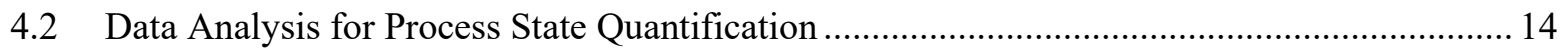

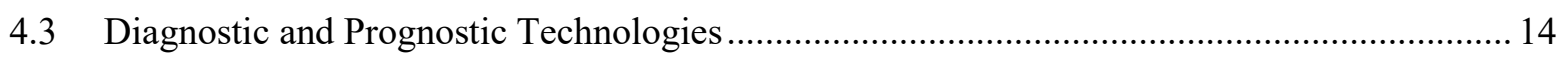

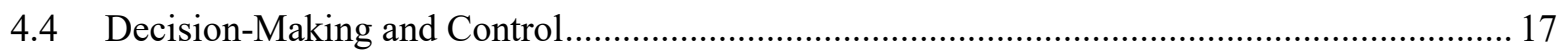

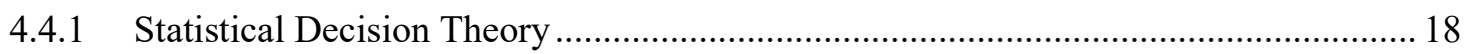

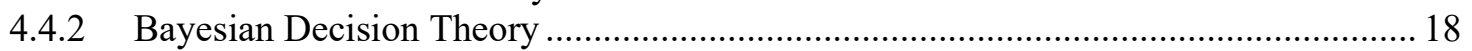

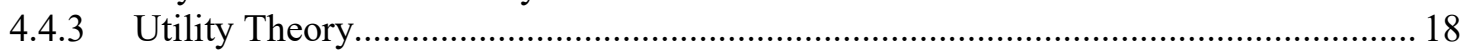

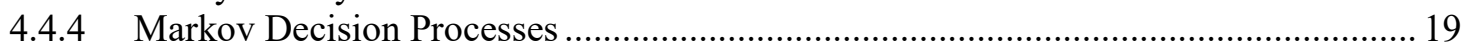

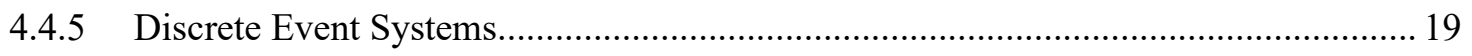

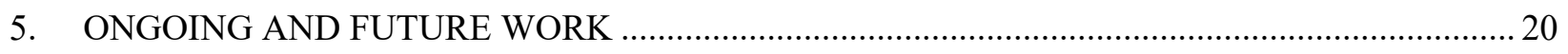

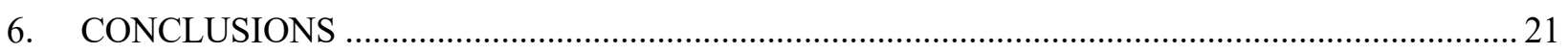





\begin{abstract}
Microreactor concepts have seen increasing interest in recent years given their potential use for meeting energy needs in remote and grid-isolated communities. Because of the small power outputs of microreactors and the potential for remote siting, there is a need to significantly reduce operational staffing levels to improve their economic viability. Autonomous control capabilities emerge as a key enabling technology.

Autonomous control should not be perceived as a single feature that when added delivers certain functions. On the contrary, autonomous control capability is a highly complex systems engineering process that requires detailed understanding of system dynamic response as a function of operational actions. These advanced capabilities can be achieved at different levels of autonomy, which would then require different levels of human operator involvement in operational decision making. The division of functions and responsibilities between the human operator and the automatic system is the starting point in the system engineering life cycle process. Once this division of functions and responsibilities is established, several options exist for autonomous control ranging from simple automation of some procedures to fully autonomous operational mode with automatic decision-making and execution without the involvement of a human operator.
\end{abstract}

A critical aspect of autonomous decision-making is the ability to have as complete an awareness of the system state as possible. In addition to monitoring the conventional process data, such as temperatures, pressures, flow, and neutron flux, measurements pertaining to the condition of important components become as important as the operational data. This type of information will allow the autonomous control system to adapt its internal configuration to changing conditions of structures, systems and components of importance through the decision-making process. The key capability is to execute these advanced functions with minimal operational disturbance and, of course, without compromising the public health and safety.

Several technical and regulatory advances are needed before widespread use of autonomous control in microreactors. These include sensor and instrumentation technologies that are capable of long-term unattended operation in harsh environments, technologies for inferring the state of the microreactor system or subsystems with confidence, algorithms for predictive decision-making that account for the assessed condition of the microreactor subsystems as well as the potential impact on those components of any operational decisions, and actuators and control system hardware that are resilient in harsh environments. In addition, given the need to remotely monitor the operations, cybersecurity requirements will likely need to be imposed to ensure secure operations.

\title{
1. INTRODUCTION
}

Microreactor concepts have seen increasing interest in recent years given their potential use for meeting energy needs in remote and grid-isolated communities. Microreactors, which are the smallest variation of small modular reactors (SMRs), are colloquially defined as those nuclear systems with design power outputs between 1 and 20 MWe. This class of reactors are conceived to serve in several capacities including as backup generators, mobile electrical sources, and heat sources for process heat applications. Cogeneration is also a possibility, though there do not appear to be any designs specifically targeting this. 
These types of small reactors are not new and were explored for base power and as mobile sources of electrical generation as early as the $1950 \mathrm{~s} .{ }^{1}$ In some instances, small reactors were considered to be stepping-stones (technology demonstration units) to larger plants of the same design. The United States at one point had up to 14 commercial reactors with capacity under $100 \mathrm{MWe}^{2}$ in addition to a number of demonstration and special purpose reactors that were built and demonstrated by the US Army. ${ }^{3}$

Most current microreactor concepts have focused on high-temperature operation (typically in excess of $500^{\circ} \mathrm{C}$ ) to take advantage of higher thermal efficiencies compared to commercial light-water reactors (LWRs). ${ }^{4}$ One consequence of higher operating temperatures is the need to adopt non-light-water coolants (e.g., helium gas). Being designed for mobility, most microreactor concepts are also physically small, and therefore, provide limited space for mounting instruments to monitor primary system components and process variables. Moreover, these concepts, particularly those targeted for deployment in remote locations, are likely to have longer operating cycles between refueling and maintenance. ${ }^{5}$

A number of challenges are associated with higher-temperature operation of microreactors, including the need to quantify strain and temperature field data for verifying design performance, demonstrate structural integrity of the primary and secondary system components, and develop new sensors and instrumentation to provide the necessary information for monitoring and controlling system performance.

A more urgent issue is the potentially unfavorable economics of any microreactor concept. Specifically, considering the small power outputs of the microreactor concepts under consideration, there is loss of economies of scale compared to larger units. ${ }^{6}$ This is not a new concern and has been an issue for smallsize reactors for a long time, ${ }^{1}$ with the renewed interest in SMRs bringing this issue to the fore in recent years. However, recent studies ${ }^{7,8}$ also note that economic analyses purely based on economies of scale may misrepresent the scale of the issue and that small reactors may be cost-competitive when other factors (for example, the specific markets, scaling of power output, and the learning rate $^{6}$ ) are all taken into account.

Of the many factors that contribute to the economics of microreactors, the controllable costs are primarily driven by the operations and maintenance $(\mathrm{O} \& \mathrm{M})$ costs. With microreactors, the O\&M costs are expected to be significant given the potential remote siting and the O\&M challenges that may arise as a result. An additional factor is the potential need to reduce the number of on-site field operators; remote operations

\footnotetext{
${ }^{1}$ W. R. Corliss, Power Reactors in Small Packages, US Atomic Energy Commission Technical Information Booklet, October 1968.

${ }^{2}$ https://pris.iaea.org/PRIS/CountryStatistics/CountryDetails.aspx?current=US.

${ }^{3}$ L. Suid, The Army's Nuclear Power Program: The Evolution of a Support Agency, Praeger, 1990.

${ }^{4}$ A. Peakman, Z. Hodgson, and B. Merk, “Advanced micro-reactor concepts,” Progress in Nuclear Energy, 107, 61-70. doi:https://doi.org/10.1016/j.pnucene.2018.02.025

${ }^{5}$ Nuclear Energy Institute, Roadmap for the Deployment of Micro-Reactors for U.S. Department of Defense Domestic Installations, Technical Report, October 2018.

${ }^{6}$ T. Roulstone and Z. Guan, "Economies of scale vs economies of volume - LWRs," Presented at the Nuclear Institute - SMR Conference, September 2014.

${ }^{7}$ M. Carelli et al., "Economic features of integral, modular, small-to-medium reactors," Progress in Nuclear Energy 52, pp. 403414, 2010.

${ }^{8}$ S. Boarin, G. Locatelli, M. Mancini and M. E. Ricotti, "Financial case studies on small- and medium-size modular reactors," Nucl. Technol., Vol. 178, pp. 218-232, 2012.
} 
capability is being considered as a result. A final factor in the cost-competitiveness is the capacity factor, with high capacity factors likely needed to maintain cost competitiveness. ${ }^{9}$

Reducing the number of required operators (both control room and field operators), the potential deployment scenarios for remote operations, and the economic pressure to maintain high capacity factors point to a need to seek for novel technologies that support operations. The gap that will arise in operational support due to the reduction of staffing level must be properly identified and addressed. To compensate for the tasks conventionally performed by human operators, more information about the process and key structures, systems and components will be useful to increase awareness about the system.

Incorporation of technologies for increasing the level of autonomy can potentially accelerate decisionmaking, which is expected to be important for flexible operations. For instance, many microreactors are expected to support generation of electrical power on-demand in contrast to relatively steady baseload power output in the commercial LWR fleet. Managing electrical power output through fast turbine runback or switching between electrical-load-dominated vs. process-heat-dominated operational mode will impose increased burden on the operators. This burden can be reduced and switching significantly facilitated through increased automation, particularly through adoption of dynamic decision-making capabilities that can handle off-normal conditions or unanticipated upsets. Due to more frequent cycling of active components, it is natural to expect a higher frequency of occurrence of upset conditions due to the higher frequency of demand changes. Through acquisition of higher density data and faster sampling, it may be possible to detect equipment performance degradation and potential upset conditions early on such that compensatory actions, or at least, mitigatory actions can be taken before they lead to major downtime. In addition, the ability to detect equipment performance degradation in a timely fashion will also assist in avoiding unnecessary maintenance activities conducted according to a time-based schedule. The result of autonomous decision-making and control actions is therefore the potential for improved economic competitiveness due to personnel costs reduction, high capacity factors, and avoided unnecessary maintenance. These decision-making functions and control actions must be executed within a designated safety envelope.

This document discusses autonomous operation concepts for microreactors, discusses initial requirements for these systems, provides examples of concepts of autonomy in reactors, and identifies desired technical advances to enable autonomous operations.

This report does not examine licensing bases for autonomous operation of reactors; that topic was the subject of a separate report. ${ }^{10}$

\section{AUTONOMOUS OPERATION OF MICROREACTORS: REQUIREMENTS}

Autonomy requires that a system possess the self- and environmental awareness attributes, and that it can adapt to new conditions to meet its mission goals. A critical component of autonomy is decision-

\footnotetext{
${ }^{9}$ M. Nichol, "Micro-Reactors," Presentation at GAIN-EPRI-NEI-US NIC Micro-Reactor Workshop, June 2019. Available online at https://gain.inl.gov/SiteAssets/Forms/AllItems.aspx?RootFolder=/SiteAssets/MicroReactorWorkshopPresentations/Presentations\&FolderCTID $=0 \mathrm{x} 012000556 \mathrm{C} 9 \mathrm{CD} 01 \mathrm{~B} 43 \mathrm{BF} 458916 \mathrm{~A} 221 \mathrm{~A} 352 \mathrm{~F} 303 \& \mathrm{View}=\%$ 7bE 4342CCA-6E12-4688-8297-617F701D2BA2\%7d

${ }^{10}$ R. J. Belles and M. D. Muhlheim, Licensing Challenges Associated with Autonomous Control, ORNL/SPR-2018/1071, December 2018.
} 
making, ${ }^{11}$ where the system has the ability to "reason" about its internal state, the environment, and its mission needs to determine a suitable change in its configuration.

Algorithms for autonomous operation and control are often the result of a systems-engineering process that defines and delineates the expectations and functions of the autonomous system relative to a human operator who may oversee the system operation. ${ }^{11}$

\subsection{DEGREES OF AUTOMATION}

The degree of automation is often described as a continuum ${ }^{11,12}$ (see Figure 1), with the level of human intervention decreasing as the system becomes more autonomous. As discussed in Cetiner et al., ${ }^{11}$ the likelihood and potential consequences of machine error increase with the level of autonomy. However, the potential for human intervention can decrease with increasing level of autonomy. Therefore, the degree of autonomy is a design decision that must be determined as a trade-off between staffing reduction, operational flexibility, system complexity, and potential safety and reliability implications.

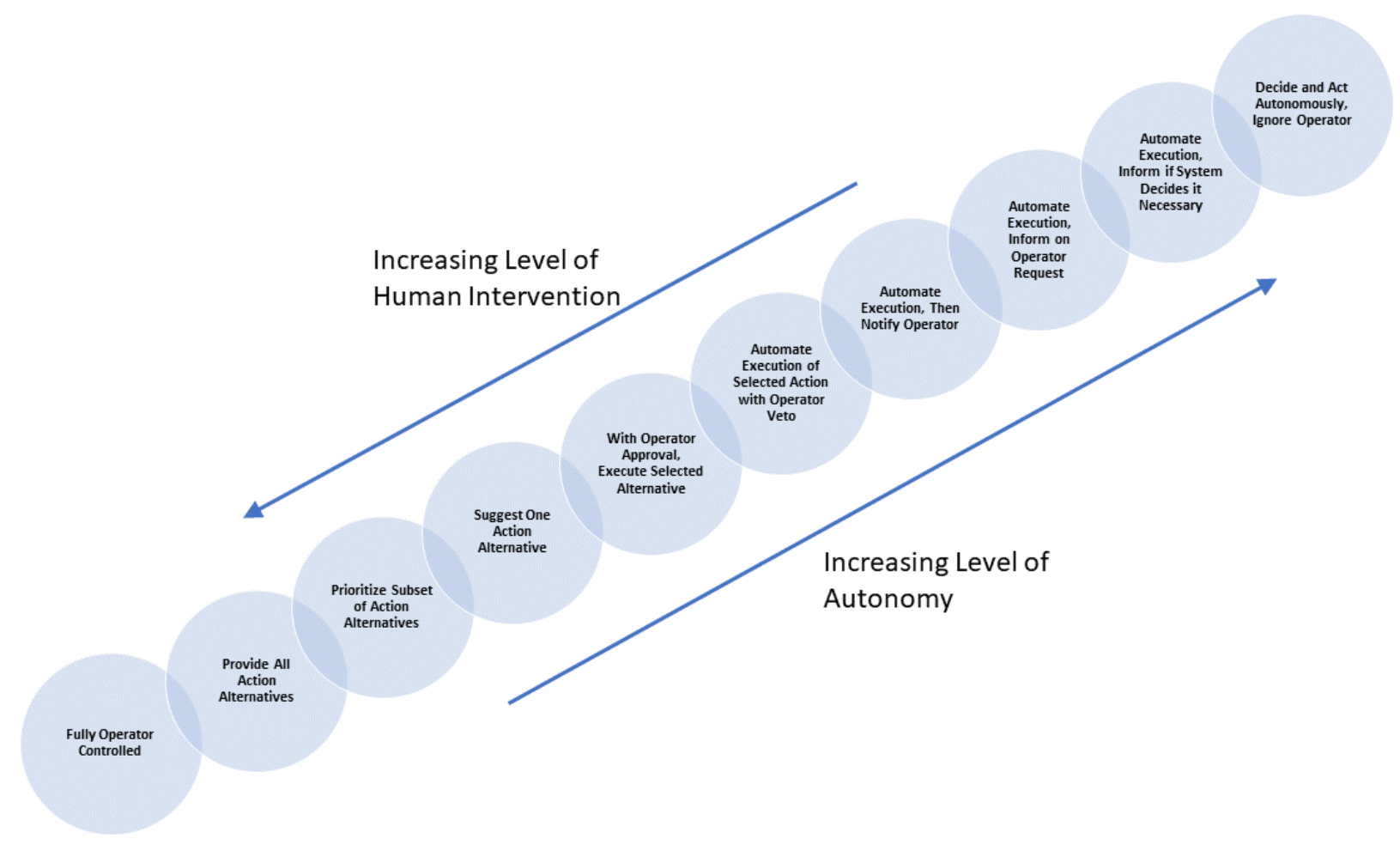

Figure 1. Degrees of automation (based on Sheridan).

\footnotetext{
${ }^{11}$ S. M. Cetiner, P. Ramuhalli, Transformational Challenge Reactor Autonomous Control System Framework and Key Enabling Technologies, ORNL/SPR-2019/1178, Oak Ridge National Laboratory (May 2019).

${ }^{12}$ T. B. Sheridan, Telerobotics, Automation, and Human Supervisory Control, The MIT Press, Cambridge, Massachusetts (1992).
} 


\subsection{MOTIVATION FOR AUTONOMOUS OPERATION OF MICROREACTORS}

Autonomous operation technology has been widely researched and is being deployed in many applications for control of complex systems. Most autonomous control architectures tend to use one or more sets of rules (if-then logic) and/or AI algorithms, along with models of the system behavior, for decision-making. An overview of applications of autonomous control is provided in Cetiner et al. ${ }^{11}$ and points to an increasing level of adoption of this technology in unmanned mobility and transportation systems.

Within nuclear power plant operations, the state of the art appears to be feedback control systems that can match electrical generation to demand through set point tracking and automating reactor and feedwater control. However, the system still requires human intervention in the event of an off-normal condition in one or more of the systems. Note that these automated control systems are separate from the reactor protection systems (RPSs) that automatically actuate when unsafe conditions are sensed.

While such automated systems (with their reliability and stability) appear to be sufficient for most commercial plants, advances in autonomous operation will be required for microreactors. As discussed previously, most microreactor concepts

- Are conceived for mobile and remote siting.

- Are designed for higher temperature operation.

- Use non-light-water coolant (such as helium gas) and may use nontraditional concepts such as heat pipes for heat removal from the core.

- Will use emerging fabrication techniques that use advanced manufacturing concepts.

- Target a wide range of applications including electrical generation supporting microgrids, backup electrical power, process heat, and nontraditional energy-intensive applications.

The following three factors drive the need for autonomy in microreactor concepts.

- Economics of operation. The potential for remote siting or use as a mobile power unit will necessitate minimizing staffing for operations. One consequence of this need to minimize staffing is the need to deploy technologies for automating plant operation as much as possible, with autonomous operation (where the decision-making is also delegated to the control system) preferable. Also, as discussed previously, increasing and maintaining capacity factors is likely to be necessary for cost competitiveness with other options (such as diesel generators) in remote locations.

- Response time. The need to potentially switch operating modes quickly in response to changes in demand or changes in conditions requires a level of automation that is currently not used in nuclear power plants. While the response time requirement is limited by the dynamics of the nuclear plant system, there is still a need for rapid decision-making and potentially incorporating demand forecasts into the process. While operator-based decision-making remains a distinct possibility, this places further demands on a potentially small number of staff, making timely decisions difficult. The alternative is to increase the number of operations staff, which runs contrary to the demands of remote siting and mobility.

- New reactor concepts. Microreactor concepts range from gas-cooled reactors to more complex systems that use nontraditional means for heat removal. ${ }^{13}$ The resulting demands on control

${ }^{13}$ P. McClure et al., Design of Megawatt Power Level Heat Pipe Reactors, Los Alamos National Laboratory, LA-UR-15-28840 (2015). 
systems require understanding the state-space of the system and identifying conditions that promote stability vs. conditions that could lead to safety concerns. Given that the state-space of these systems can be large compared to traditional concepts, economics may dictate the development of responses to most-likely scenarios, with responses to other potentially high-consequence but unlikely events delegated to an autonomous system. Operation of the plant at elevated temperatures also brings the potential for accelerated degradation in some components; economic viability will require the ability to quickly and autonomously respond to changing conditions in the plant.

Among the enabling technologies for autonomous operation and control are sensors for process and system state monitoring, computing systems for real-time data analysis, and algorithms for information fusion and decision-making. Recent advances in each of these areas are enabling wider deployment of autonomous systems. For instance,

- Advances in sensors and circuit design have led to cheap wireless sensors for monitoring parameters. ${ }^{14}$ This in turn enables the monitoring of parameters that may have been too expensive to monitor in the past.

- Advances in computing hardware are allowing larger data sets to be held and processed in memory. This enables faster data analysis as well as the potential for fusing information from more sources than in the past. ${ }^{15}$

- Advances in algorithms for machine learning (ML) and information fusion are enabling development of new approaches for analysis of data and the extraction of greater levels of information than in the past. Again, this enables greater insights into the system state and helps inform decision-making.

\subsection{INITIAL HIGH-LEVEL REQUIREMENTS FOR AUTONOMOUS OPERATION}

Within the context of nuclear plant systems and specifically microreactors, full autonomy of operation and control will likely require the following: ${ }^{11}$

- Implementation as a non-safety-related system. The implementation will need to meet all applicable rules and regulations regarding separation between and isolation of safety- and nonsafety-related systems and noninterference with any safety-related function.

- Compatibility with the reactor concept of operations. The concept of operations which specifies the functional separation and interfaces between the autonomous system and the human operator.

- Operator interaction. Ability to accept operator commands and incorporate and appropriately prioritize those commands along with other control decisions.

- Autonomous decision-making capability. Autonomous decision-making capability will need to function over multiple operational modes. This is a step up from normal automation of operations and control, with predefined parameter ranges.

- Short response times. Note that the reactor system dynamics will need to be defined to specify acceptable response times for decision-making.

- Long-term reliability of the autonomous system. This high-level requirement ensures that degradation of systems, structures, and components (SSCs), including the sensors and

\footnotetext{
${ }^{14}$ M. CW. Kintner-Meyer, “Opportunities for wireless sensors and controls for building operation,” Energy Engineering, 102(5): 27-48, 2005. doi:10.1080/01998590509509441

${ }^{15}$ D. Raheja, J. Llinas,, R. Nagi, and C. Romanowski, "Data fusion/data mining-based architecture for condition-based maintenance," International Journal of Production Research, 44(14), 2869-2887, 2006. doi:10.1080/00207540600654509
} 
instrumentation that are part of the autonomous operation system, has minimal impact on the safety and operation of the plant. Achieving this may require redundant systems as well as technology to quantify degradation and correct for it.

- Determinism and causality. This requirement enforces the condition that the decision algorithm generates the same output for a given set of inputs. In other words, the internal state or the memory of the decision algorithm should not have an impact on the output.

- Robustness of decisions and ability to handle uncertainties. Uncertainties in measurements are expected and typically arise as a result of sensor drift; degradation or failure; or, more importantly, insufficient coverage of the quantity being measured. This requirement ensures that these uncertainties are properly taken into account in the decision-making algorithm.

- Testability. This requirement ensures that the decision-making algorithm can be tested with a high test coverage that meets the system reliability and operational availability goals. Additionally, these tests must ensure that unanticipated transitions that violate the safety rules and operational principles are blocked.

- Explainability of decisions. This requirement, if addressed, provides a mechanism for documenting the reason for each decision. This provides traceability and will be an important factor in building trust in the autonomous operations and control system.

- Security. This requirement is imposed by the potential for remote siting of microreactors. Remote operations impose a need for communications between the reactor instrumentation and controls $(\mathrm{I} \& \mathrm{C})$ and some central monitoring facility. Cybersecurity requirements on the communications channel facilitate securing the data/information flow across the channel and limit the ability of a malicious actor to damage the reactor system.

\section{AUTONOMOUS OPERATION CONCEPTS}

\subsection{GENERIC FRAMEWORK FOR AUTONOMOUS OPERATION}

A generic sequence of steps for enabling autonomous operation is shown in Figure 2. Fundamentally, any autonomous system will need to be capable of measuring observables and quantifying system state so as to allow an assessment of the condition of the system. The information will then be applied to infer whether the present state is able to meet the current demand profile and the anticipated (projected) demand profile. Using either models (which can be computationally expensive) or other decision-making methods (including ML), the autonomous system then identifies alternative allowable states that (1) meet safety and reliability requirements and guarantee, to the extent possible, sustaining the operations, (2) are reachable in a timely manner using allowable state transitions, and (3) are capable of meeting the anticipated demand profile by the time the system gets there. These high-level decisions are then executed by the lower level control systems through conventional actuation signals to slowly navigate the system from the current state to the desired state. 


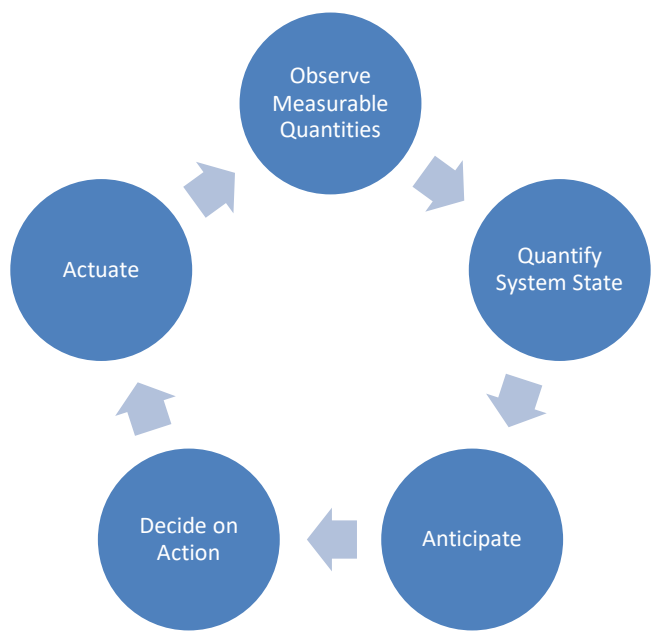

Figure 2. Generic sequence of operations for autonomous systems.

Clearly, this high-level description overlooks several details that are critical to proper function of the autonomous operation of the microreactor. A key complication arises from the fact that it is virtually impossible to have full information about the system state; that is, the state of the process as well as the condition of key components. This shortcoming obviously results from the inability to fully instrument a system to obtain all key information and fully observe the health of critical assets. Therefore, a key attribute of decision-making should be robustness against lack of full knowledge due to limited data and uncertainties in measurements.

The inference and the decision-making can be performed using either a purely deterministic method, purely risk-informed method, or some combination of the two. Deterministic methods are those that capture the system behavior through the governing dynamics. They typically use mechanistic models that cannot fully capture small-scale behavior, which, for certain complex physical behaviors, may be critical on the macroscopic scale. Additionally, purely mechanistic models are typically generated through a set of assumptions that, under certain conditions, may be violated. Moreover, they may also have unmodeled dynamics. In other words, purely mechanistic models, despite the advent of modern computational methods and compute platforms, may not fully capture the entire domain of a complex system behavior. While the uncertainties associated with principal states and the sensitivity of the system behavior can be investigated, these computations may also be costly.

Risk-based and risk-informed methods, on the other hand, exploit the concept of risk associated with the implementation of decisions. Conventionally, risk is defined as follows:

$$
\text { Risk }=\text { Probability } \times \text { Consequence } .
$$

The advantage of this approach is to identify key consequence states, ideally associated with the probability distribution that defines the likelihood of these consequences. This approach may enable more efficient generation of strategies, based on the prior knowledge of failure modes (and effects) or degradation modes, given detection of an upset condition. Risk-informed methods can also help identify strategies that minimize economic risk metrics while ensuring that safety margins are not compromised. Once a down-selection of "reasonable" options is identified, a more detailed computation of mechanistic consequence analysis may be achievable.

Within the context of decision-making, the control system design depends on the reactor design. Many of the proposed microreactor concepts appear to have fairly slow dynamics and tend to be relatively 
self-controlling (i.e., large negative coefficients of reactivity with increasing temperature, Doppler broadening, etc.). Under these conditions, it is likely that the primary function of the autonomous system will be to ensure that reactor operations do not drift into states that if left uncorrected may impact safety. In most instances, stability analyses in the frequency domain, such as Nyquist criterion or Bode gain- and phase-margin calculations, ${ }^{16}$ provide ample information and confidence about the stability of the system. For nonlinear dynamics, it may be necessary to perform frequency-domain analysis at various equilibrium points of the system, such as different power levels. Moreover, for systems whose dynamics can be adequately represented analytically, Lyapunov stability analysis can be performed. ${ }^{17}$ However, identifying a Lyapunov function can be challenging even for a simple system; and it is significantly more so for large-scale complex systems.

Another critical element in designing a near-autonomous system is the clear delineation of tasks and responsibilities between humans and automation. These responsibilities are captured in a formal analysis, which is typically referred to as the human-automation collaboration. ${ }^{18,19}$ This document essentially establishes the level of automation, as shown in Figure 1, and forms the basis for all design activities, including the control system design, and the component sensing and actuation interface definitions.

The following are examples of autonomous microreactor operations at different degrees of automation.

- Fully manual operation and control. Every input from sensors is fed to the operator for decision-making and actuation. While not really autonomous, this is a baseline against which the performance of any autonomous system can be compared.

- Automated procedures. Limited decision-making by the controller, with manual selection of mode changes.

- Reactive control system (RCS) only decision-making. Limited to autonomy within a single operation mode. Some level of run backs may be used to manage operations. The RPS is independent and supersedes RCS action if trip set points are exceeded.

- Risk-informed decision-making. Autonomous operation decision-making includes some mode transitions. Risk-informed decision-making attempts to keep systems within predefined operational behavior boundaries. This approach can be reactive (not very useful) or predictive (which is more useful). The predictive approach needs to incorporate demand predictions and plant condition estimates and predictions.

With the exception of fully manual operation and control, technology development and maturation is essential for deploying autonomous control and operation with any degree of automation.

\footnotetext{
${ }^{16}$ K. J. Åström, R. M. Murray, Feedback Systems, Princeton University Press, Princeton and Oxford (Oct. 2016).

${ }^{17}$ Given a nonlinear system $\frac{\mathrm{dx}}{\mathrm{dt}}=\mathrm{F}(\mathrm{x})$, let $\mathrm{V}$ be a function, and $\dot{\mathrm{V}}$ be time derivative of $\mathrm{V}$ along the trajectories of the system dynamics. If there exists $r>0$ such that $V$ is positive definite and $\dot{V}$ is negative semidefinite on $B_{r}$, then the system is said to be stable in the sense of Lyapunov. If $V$ is positive definite and $\dot{V}$ is negative definite on $B_{r}$, then the system is asymptotically stable.

${ }^{18}$ R. C. Johnson, et al., "Autonomy and Automation Reliability in Human-Robot Interaction: A Qualitative Review." Proceedings of the Human Factors and Ergonomics Society Annual Meeting, 53(18), 1398-1402, 2009. doi:10.1177/154193120905301849.

${ }^{19} \mathrm{C}$. Zhang, et al., "Human-centered automation for resilient nuclear power plant outage control," Automation in Construction, 82, 179-192. doi:https://doi.org/10.1016/j.autcon.2017.05.001.
} 


\subsection{TRANSFORMATIONAL CHALLENGE REACTOR FOR DEMONSTRATION OF NEAR-AUTONOMOUS OPERATION}

This section is intended as a summary; further information can be found in Cetiner and Ramuhalli. ${ }^{11}$

The key driver for the transformational challenge reactor (TCR) I\&C system design is minimization of potential regulatory challenges for the implementation of the I\&C platform. This will be accomplished by relying on approved components and systems that have safety or safety-related functions - to the extent possible. For non-safety-related controls (i.e., the plant control system and other ancillary controls), more advanced control approaches can be adopted if they have sufficient industrial pedigree.

For the present application, autonomous operation focuses on moving the following human functions to algorithms:

- Step change in power level (increase or decrease) in response to an increase in demand and

- Slow ramp between two steady state operation modes.

\subsubsection{Hierarchical Architecture}

TCR will adopt a three-layer control architecture, as shown in Figure 3.

The functional layer of the TCR autonomous control system architecture includes sensors, actuators, and feedback control systems to perform the low-level control functions. This layer uses a conventional I\&C system architecture designed to deliver reasonable stability and a nominal set point tracking performance in the event of an anticipated operational occurrence.

The TCR I\&C system provides the hardware, software, and data communication infrastructure to enable the sensing, monitoring, processing, actuation, and protective functions of the plant.

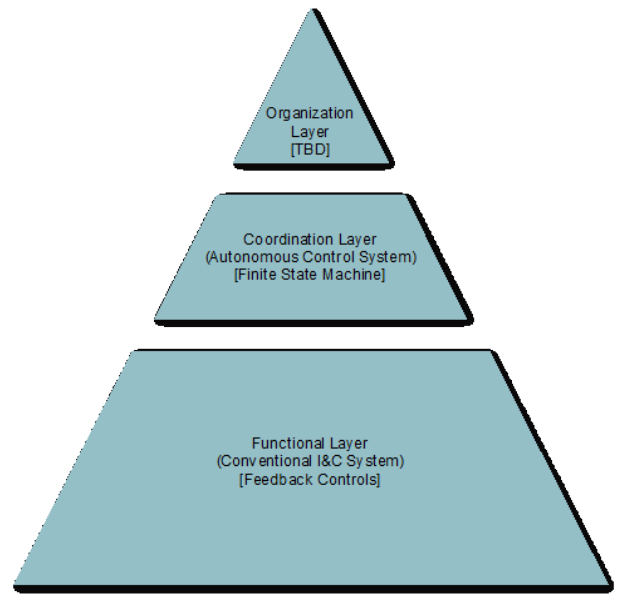

Figure 3. High-level architecture of the transformational challenge reactor autonomous control system. ( $\mathrm{I} \& \mathrm{C}=$ instrumentation and controls; $\mathrm{TBD}=$ to be determined.)

Conceptually, the TCR I\&C architecture is organized in two major divisions: 
1. The conventional plant I\&C system responsible for the protection and control functions; these are essential sensing and actuation functions that provide monitoring of key safety and performance variables and that generate reactor trip and plant control signals.

2. Extended monitoring to enable acquisition of data from key SSCs.

The coordination layer of the TCR autonomous control system includes sensors, actuators, and control system hardware and software that implement the finite state machine - also called finite state automaton - to perform higher level control functions. Unlike the continuous-time-domain actuations performed in the functional layer, the control functions in this layer are intended for major operational changes such as transition from one mode of operation to another. Operating modes and the associated mode transitions are the standard means of achieving plant start-up and shutdown. These modes and transitions are highly proceduralized, labor-intensive (in large nuclear power plants), and time-consuming activities.

Targeting higher level control functions with a scope on mode transitions is useful for two reasons:

1. It provides a vehicle for demonstrating the autonomous control functionality, and

2. It provides a reasonable scope for the demonstration of autonomy without creating undue technical risks and potential regulatory risks for the TCR program.

A discrete event system (DES) was chosen to model the behavior of a rod control system and the requirements of a reactor start up because the system can easily be expressed as finite state automata (FSA), and their properties can be shown using formal proofs. The two properties to be noted are non-blocking and controllability. An automaton is non-blocking if the end or marked state is reachable from every attainable state, showing that there are no dead ends, or infinite loops with no way to break them. Controllability is a property of the languages generated by two automata, and states that a supervisor's language is controllable with respect to a plant's language only if any uncontrollable events found in the plant's language also occur in the supervisor, showing that the plant cannot uncontrollably move to a state that is not within the supervisors state space. ${ }^{20}$

The scope of desired semi-autonomous operation was limited to startup from hot-standby to full power, and the ability to follow a power profile during the mission of the system. The critical systems and subsystems required to execute a startup need to be modeled using FSA to demonstrate the non-blocking and controllability properties.

Specifically, the goal is to model a generic rod control system that captures all the needed elements of rod motion found in all reactors without becoming overly specific to a certain design's addition restrictions, and to provide enough complexity to make the proofs meaningful. To achieve this, the assumed system will consist of three groups of rods with the ability to give a motion command of STOP, INSERT, or WITHDRAW to the selected group. Once the rod selection and rod movement process are individually modeled, and then parallel composition is used to create the overall plant model. The procedure for selecting and moving rods to the point of criticality is also modeled as an FSA.

FSAs are denoted by a five-tuple in the following form

$$
G=\left(X, E, f, x_{0}, X_{m}\right)
$$

\footnotetext{
${ }^{20}$ C.G. Cassandras, S. LaFortune, Introduction to Discrete Event Systems $2^{\text {nd }}$ edition, Springer Science+Business Media, New York, New York (2008).
} 
where $X$ is the set of states, $E$ is the set of events associated with $G, f: X \times E \rightarrow X$ is the transition function that maps a state and an event to another state, $x_{0}$ is the initial state, and $X_{m} \subseteq X$ is the set of marked state. FSAs can also be represented as a transition diagram, with the states as boxes, transitions as arrows between the states, events as labels on the transitions, the initial state denoted by a loose arrow, and the marked states by a double border on the state.

The organization layer deals with more complex decision-making algorithms that rely primarily on dynamic search-based methods. Currently, this option is not considered within the scope of TCR autonomous control.

\section{AUTONOMOUS OPERATION OF MICROREACTORS: POTENTIAL APPROACHES AND DESIRED TECHNICAL ADVANCES}

As discussed previously, the degree of autonomy depends on the reactor design and concepts of operation. However, all autonomous control systems should address two concurrent missions relative to the reactor:

- Safety. This is a prerequisite and requires the ability to maintain $\mathrm{k}_{\text {eff }} \sim 1$ and continuous removal of heat to prevent core meltdown. Passive safety systems are generally inherent to the microreactor system design and require no external actuation. However, some concepts also include safety systems that require active control. Many design concepts invoke feedback, doppler broadening, etc., and provide for simplifying control system design.

- Operability. This is the ability of the reactor to meet time-dependent demand needs. Examples include load following, and switching between or simultaneous support of electrical and process heat. The autonomous operation and control system will need to be able to manage a wide range of transitions to navigate from one mode of operation to another.

To meet these broad missions, the autonomous control system will need technologies that span the observability and controllability domains, including the following.

- Sensors and instrumentation

- Sensors and instrumentation compatible with a harsh environment for measuring key operational parameters

- Systems to provide power to sensors/instrumentation and data telemetry technology for data exfiltration and transmission

- Measurement sensors and instrumentation for online monitoring (OLM) and control of equipment and structural state and actuator positions

- Diagnostic methods for quantifying process and equipment state, including OLM technologies for fault detection in systems and fault detection in measurement instrumentation

- Prognostic technologies for predictive assessment of component conditions

- Technologies for decision-making regarding operating mode selection and control response (includes system models)

\subsection{SENSORS AND INSTRUMENTATION}

Measurement data indicating the state of the system are critical for autonomous control decision-making. The system state for a reactor is often derived from measurements of temperature, flow rate, neutron flux, 
and pressure at different points in the primary and secondary systems. These parameters provide the data for heat balance calculation and are inputs into the control logic.

However, for autonomous operation, additional parameters are likely needed to assess system state. These include data on the condition of the SSCs, including the structural health of primary system components such as the heat exchanger and the mechanical condition of any actuators. The specifics depend on the reactor and operational concept. For example, a tightly coupled system such as a heat-pipe-based high-temperature reactor is likely to require information on heat-pipe failures and thermal strain in the primary system to be able to assess whether the heat-removal system is adequate. On the other hand, the need to monitor strain in the reactor core may not be as urgent in conventional concepts where a working fluid flowing through the core is used for heat removal.

However, most microreactor concepts have small cores that are not ideal for deploying a dense set of sensors for long-term monitoring at high temperatures. Therefore, technical advances for sensors and instrumentation to enable autonomous control will need to include the following.

- Ability to tolerate the harsh operating conditions in microreactors. Anticipated conditions include high temperatures $\left(>550^{\circ} \mathrm{C}\right)$, corrosive coolant media, and fast neutron spectra (in some designs).

- High sensitivity, to ensure that the measurements provide the necessary level of granularity for use in control and operational decision-making.

- High reliability to ensure measurement quality does not suffer over the lifetime of the microreactor. Methods such as online calibration for ensuring continued reliability of the measurements through the lifecycle of the microreactor may also be necessary.

- Ability to tolerate and operate in the limited physical space in microreactors (referred to as "deployability"). As a consequence of the limited space and access, methods for embedding some sensors within the core or heat exchanger-heat transfer system may be necessary for obtaining adequate information. Alternatively, methods for inferring the necessary information from a limited set of measurements may be needed. While such "virtual sensors" have been proposed for nuclear applications ${ }^{21}$ and been used in a limited form elsewhere, ${ }^{22,23}$ we are not aware of any deployed virtual sensors in nuclear power plants.

- Short response times to ensure that measurements of system state track changes in the state with a reasonable time lag. Acceptable response times are likely to depend on the specifics of the reactor concept and its dynamics.

Several existing sensor technologies are likely to be applicable, meeting the requirements for instrumenting most currently proposed microreactor concepts. These technologies include thermometers, flow meters, strain sensors, neutron and gamma sensors, and heat flux sensors based on a variety of measurement physics, ${ }^{24}$ though several of these technologies remain at a lower technology readiness level and need further development and testing. Their applicability for embedded sensing also remains to be

\footnotetext{
${ }^{21}$ Tipireddy R, ME Lerchen, and P Ramuhalli. 2017. "Virtual sensors for robust on-line monitoring (OLM) and Diagnostics." In Proc. ANS NPIC-HMIT 2017, San Francisco, June 11-15, 2017, pp. 719-728.

${ }^{22}$ S. Kabadayi, A. Pridgen, and C. Julien, "Virtual Sensors: Abstracting Data from Physical Sensors," in "2006 International Symposium on a World of Wireless, Mobile and Multimedia Networks," (2006), pp. $587-592$.

${ }^{23}$ T. Jin, Z. Fu, and G. Liu, "Data Mining for Soft Sensing Modeling of Power Plant Parameters," in "2009 Sixth International Conference on Fuzzy Systems and Knowledge Discovery," Institute of Electrical \& Electronics Engineers (IEEE) (2009).

${ }^{24}$ K. Korsah, P. Ramuhalli, et al (2016). Assessment of Sensor Technologies for Advanced Reactors (ORNL/TM-2016/337.
} 
assessed. In addition, it is not clear what measurements are essential for autonomous operation and which measurements might be considered necessary only for gathering operational data from a first-of-a-kind deployment.

\subsection{DATA ANALYSIS FOR PROCESS STATE QUANTIFICATION}

Measurement data from microreactors may be of process parameters (temperature, pressure, and flux) and of component condition (for instance, strain in the reactor core). All available information will need to be integrated appropriately to ensure an accurate estimate of the state of the plant. Such information fusion will require approaches that can use information from a limited number of sensors to estimate the state.

A large body of work in the area of state estimation problems is available to choose from for this purpose ${ }^{25,26}$ though data sets for use in fine-tuning the algorithms are still limited. A challenge is the limited information that appears to be available in the public domain regarding microreactor designs, operational states, and related process conditions. Data sets from test-beds will help in this regard. Alternatively, models of microreactor operation (even if these models are reduced-order models) that can quantify expected measurements for several operational states would likely be useful in further developing data analysis methods. In all instances, uncertainty propagation through the estimate is a challenge and will need to be addressed.

\subsection{DIAGNOSTIC AND PROGNOSTIC TECHNOLOGIES}

This set of technologies focuses on identifying signatures from monitoring data and applying these signatures for timely identification and quantification of incipient failure in SSCs. The diagnostic result is used to compute a health index, an indicator of the ability of the SSC to meet its functional goals. This information is important from the perspective of autonomous operation and control of microreactors as the control decision will depend on the system state and the perceived ability of SSCs to meet their functional goals. $^{27}$

Diagnostics for SSC degradation require signatures ${ }^{28,29}$ that are invariant to minor changes in system state and can account for missing or failure data. This requires sensor models and invariance transformations ${ }^{30}$ of the data for enhancing signatures and normalizing data to ensure the diagnostic algorithms are able to generalize across data variations. Models of normal component function ${ }^{31}$ have been proposed to identify deviations in performance and the onset of degradation that may lead to incipient failure. The ultimate goal of these techniques is a health index of the SSCs.

\footnotetext{
${ }^{25}$ Dehghanpour, K., Wang, Z., Wang, J., Yuan, Y., \& Bu, F. (2019). A Survey on State Estimation Techniques and Challenges in Smart Distribution Systems. IEEE Transactions on Smart Grid, 10(2), 2312-2322. doi:10.1109/TSG.2018.2870600

${ }^{26}$ A. Alofi et al, “A review of data fusion techniques,” Int'1. J. Computer Applications, 167(7), June 2017, pp. 37-41.

${ }^{27}$ Cetiner et al., Supervisory Control System for Multi-modular Advanced Reactors, ORNL/TM-2016/693, 2016.

${ }^{28}$ Dib, G., et al., "Ensembles of Novelty Detection Classifiers for Structural Health Monitoring using Guided Waves," Smart Materials and Structures 2017. 27(1).

${ }^{29}$ Ramuhalli, P., "Neural Network Based Iterative Algorithms for Solving Electromagnetic NDE Inverse Problems," in Electrical Engineering, 2002, Iowa State University: Ames, Iowa.

${ }^{30}$ H. Fujun and S. Wengang, "WPT-SVMs Based Approach for Fault Detection of Valves in Reciprocating Pumps," in American Control Conference, 2002. Proceedings of the 2002, vol. 6, pp. 4566-4570, 2002.

${ }^{31}$ Lee, J.K., et al. "Mathematical Modeling of Reciprocating Pump,” J Mech Sci Technol, vol. 29(8), pp. 3141-3151 (2015).
} 
The development of diagnostic methods requires reduction of uncertainties in the data and development of algorithms for signature extraction and diagnosis. Several approaches exist for uncertainty quantification and reduction, including Gaussian processes, Latin hypercube sampling, polynomial chaos methods, etc. ${ }^{32}$ and are likely to be readily transferred to application with microreactors.

Given the potential need to use measurements of the process (environment), as well as data from the SSC (for example, strain in the reactor core structure), information fusion methods may be necessary. Technical advances in data fusion for diagnostics include integration of different types of data (for instance, image data and time-series data), which encompasses the need to address specific algorithms and parameters. Other needs include algorithms for robust, automated spatial and temporal coregistration of data and accounting for differing levels of uncertainty in the different measurements. Methods to account for differing levels of uncertainty are also available ${ }^{33}$ (for instance, Khan et al. 2011), but these techniques need verification and validation before broad application.

A critical element of diagnostics is identification of conditions where the measurement system itself is failing. ${ }^{32}$ Again, this is an area of active research and development, with multiple approaches to OLM of the instrumentation available for detecting faults in the instrumentation as well as measurement drift due to sensor aging. ${ }^{34,35}$

Technologies such as prognostics health management (PHM) systems provide enhanced information on component or equipment condition, ${ }^{36}$ with predictive estimates of component failure that are customized for each microreactor unit and account for the specific configuration and operational history of the unit. PHM systems use information about operational modes, degradation accumulation models, stressors, and component history to estimate time-to-failure and remaining useful life. Figure 4 shows a schematic overview of this integration, with sensors measuring key indicators that are correlated with the condition of the components. The measurements are usually acquired on a predefined schedule (for example, once a day, or once an hour). These measurements, in conjunction with information on the local environmental conditions and models of degradation growth, are used to compute the condition of the component and provide predictive estimates of time to failure. As more measurements become available, the estimates are updated and can be integrated with risk monitors to provide predictive estimates of risk.

\footnotetext{
${ }^{32}$ Coble, J.B., et al., “Approaches to Quantify Uncertainty in Online Sensor Calibration Monitoring,” in American Nuclear Society Winter Meeting and Technology Expo, 2013.

${ }^{33}$ T. Khan, P. Ramuhalli, and S. Dass, "Particle-Filter-Based Multisensor Fusion for Solving Low-Frequency Electromagnetic NDE Inverse Problems," IEEE Trans. Instrum. Meas., vol. 60, no. 6, pp. 2142-2153 (2011).

${ }^{34} \mathrm{~S}$. Cetiner et al, Determination of sensor quality of calibration using advanced data analytics and machine learning methods, INL/EXT-19-55407 (ORNL/SPR-2019/1310), 2019.

${ }^{35}$ P. Ramuhalli, et al., Development of Fault Detection and Virtual Sensing Methodologies for Robust Online Monitoring, Technical Report, PNNL-25382, 2016.

${ }^{36}$ Coble JB, P Ramuhalli, LJ Bond, W Hines, and B Upadhyaya, “A Review of Prognostics and Health Management Applications in Nuclear Power Plants," Int'l. J. PHM, Vol. 6, 2015 (22 pages).
} 


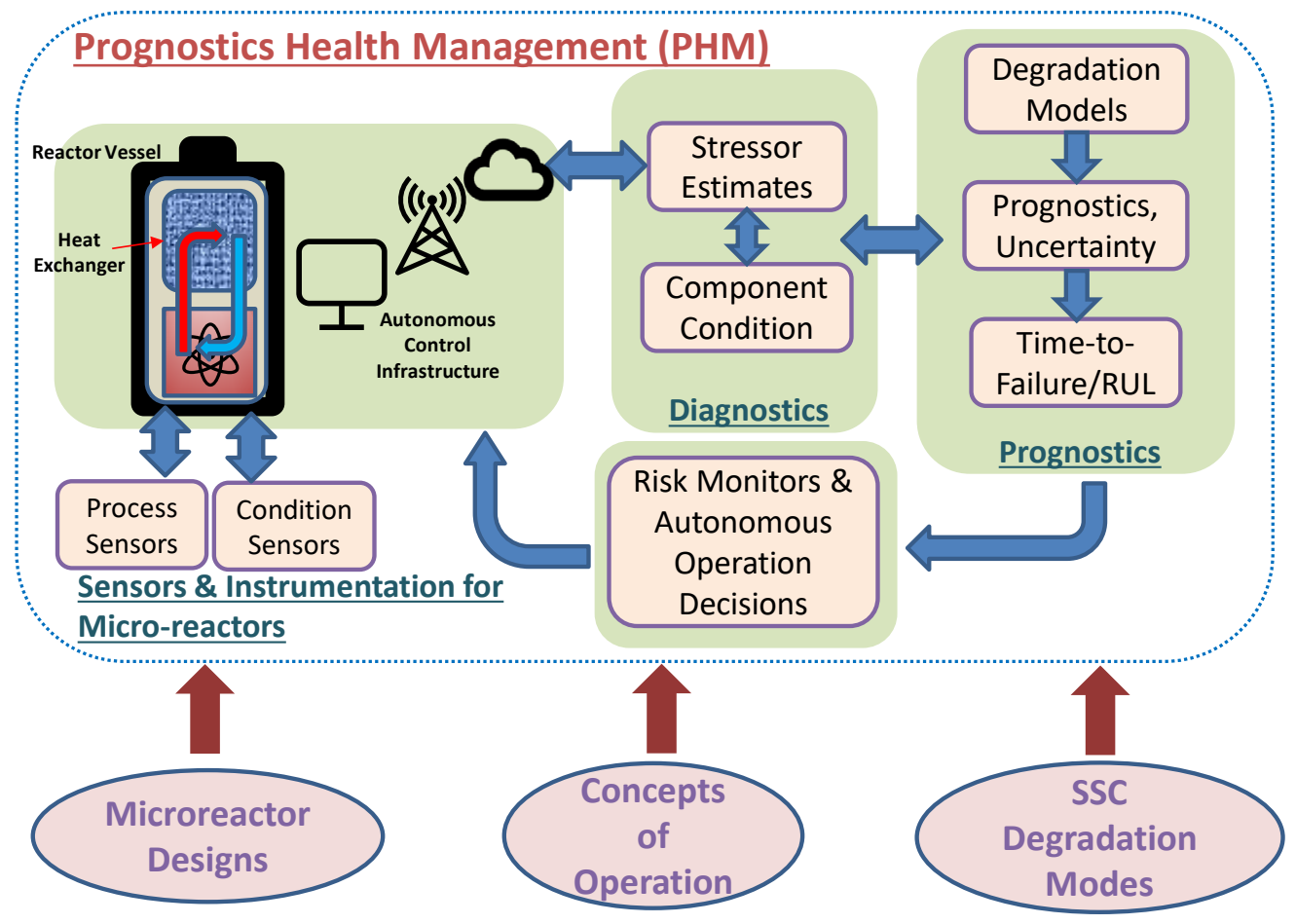

Figure 4. Prognostic system for health management (adapted from Ramuhalli et al. $\left.{ }^{37}\right)$. $(\mathrm{RUL}=$ remaining useful life; $\mathrm{SSC}=$ system, structure, and component.)

While these technologies are slowly being applied for nuclear energy systems, they have a long history of deployment in other applications (aerospace and rotating industrial machinery being the more prominent examples) as enabling technologies for predictive maintenance activities. We expect the methods that have been developed for these applications, as well as algorithms that are currently being tested with LWRs, can be readily adapted to meet the needs of microreactor component condition estimation.

For prognostic methods, a variety of algorithms are available and include sequential Markov processes ${ }^{33}$ and Bayesian networks. ${ }^{36,38}$ Both Markov processes and Bayesian networks use conditional transitional probabilities for statements about the likelihoods of different alternative future system states. While implementation details vary, in both cases the conditional probabilities of changes of SSC symptom states, based upon symptom measurements reflecting physics of failure mechanisms, are used in Bayesian updating to provide refined values of the probabilities of future system states. This process of updating the relevant probabilities continues as new data are obtained concerning the SSC symptoms. From this, the likelihoods of alternative future state trajectories can be obtained using Markov process models where the state transition frequencies are estimated based upon the symptom measurements. In both cases, these changes in state may be due to inherent system degradation or human actions and errors in response to SSC failures (mistakes in system realignments required by SSC-initiated transients or in SSC control

\footnotetext{
${ }^{37}$ P. Ramuhalli et al., Component-Level Prognostics Health Management Framework for Passive Components, Technical Report, PNNL-24377 R0, 2015.

${ }^{38}$ P. Ramuhalli, L. J. Bond, J. W. Griffin, M. Dixit and C. H. Henager, Jr., “A Bayesian prognostic algorithm for assessing remaining useful life of nuclear power components," Proc. 7th ANS NPIC-HMIT, pp. 875-886 (2010).
} 
errors). In either scenario, we expect that these changes will be indicated by deviations of sensor data from expected values.

Note that prognostic technologies are only one element of a strategy for predictive assessment in microreactors. Models simulating microreactor physics may be necessary for assessing the impact of a specific SSC condition and an explicit control action. Technical needs in this context include the following.

- Capturing the effects of coupling between components and systems. Part of the challenge appears to be limited information or models of system coupling for interconnected systems. Microreactor concepts have also been associated with variable loading and challenges current PHM methods, though concepts for microreactor application in cogeneration may help manage variations on the reactor power. ${ }^{39}$ Although some efforts have been made to combine SSC condition with variable future loading for prognosis, the work reviewed to date assumes that the future sequence of operating conditions is known. In practice, this may not be true. These approaches will need to be adapted to account for uncertain (or in some cases unknown) future loading conditions.

- Life-cycle prognostics. This relates to the ability to use different predictive models as the SSC condition changes and operational stress conditions change.

- Failure models that may be applied in remaining service life assessments for SSCs. While such models ideally capture the physics of failure associated with different failure modes, such models are more likely to be based on observed failure data. In most instances, such data-driven models are sufficient for calculating remaining life estimates as long as they are operated within the bounds of the model applicability.

\subsection{DECISION-MAKING AND CONTROL}

Autonomy should not be perceived as a plug-and-play capability. On the contrary, it is a product of a highly complex systems engineering process that clearly defines the functions expected from the control system and the allocation of such functions between the automation and human operators.

The primary gap in achieving this vision relates to decision-making capabilities that are strategic, interpretive, predictive, and adaptive. This level of self-cognition is necessary to support the vision of near-autonomous nuclear power generation. Decision-making is the foundational process that enables this capability.

Decision-making is one of the basic cognitive processes of human behaviors by which a preferred option or a course of actions is chosen from a set of alternatives based on certain criteria. Mathematically, decision-making is a problem-solving activity to identify and analyze the available courses of action and to determine the most appropriate option given the set of conditions and constraints. The solution space can differ vastly, depending on the nature of the problem being solved.

As engineering systems and processes have become increasingly more complex, with significantly higher degrees of interconnectedness, designing automation systems to address a wide range of operating conditions and equipment availability has become a daunting task. Logic tables that have been widely used to address a limited set of scenarios under nominal conditions are not comprehensive enough to cover possible scenarios as a function of various system conditions. Therefore, capabilities are needed to (1) diagnose a situation; (2) identify a viable course of actions; and (3) determine the best, optimal, or at

\footnotetext{
${ }^{39}$ IAEA, Opportunities for Cogeneration with Nuclear Energy, NP-T-4.1, 2017.
} 
least acceptable action or sequence of actions to transition to a safe state. This process is called decisionmaking.

\subsubsection{Statistical Decision Theory}

Statistical decision theory is concerned with making decisions based on statistical knowledge, which sheds light on some of the uncertainties involved in the decision problem. The field of classical statistics is directed toward using sample information arising from statistical investigation to make inferences about the use of data. In contrast, decision theory attempts to combine the sample information with other relevant information, with the intention of making the best decision.

In addition to sample information, two types of nonsample information are typically relevant. The first is the knowledge of possible consequences of decisions. Often this knowledge can be quantified by determining the loss that would be incurred for each possible decision and for various possible values of uncertainties. The incorporation of a loss function into statistical analysis was first studied extensively by Wald. $^{40}$

The second source of nonsample information that is useful to consider is called prior information. This is information about uncertainty arising from sources other than statistical investigation. Generally, prior information comes from past experience about similar situations involving similar uncertainties.

\subsubsection{Bayesian Decision Theory}

The Bayesian approach is one of the most commonly referenced mathematical methods used in decision-making processes in a wide range of applications. In Bayesian decision theory, the choice function is called a decision rule. A loss function is adopted to evaluate the consequences of an action. Using the loss function to determine possible risks, a choice function is derived for decision-making.

A generic Bayesian decision process can be divided into two phases: the inference phase and the decision phase. In the inference phase, posterior probabilities are obtained using the prior information associated with the random processes used in the decision-making process. In the decision phase, alternative decisions are identified, and an optimal decision is determined based on the construct of the loss function.

\subsubsection{Utility Theory}

Utility theory was developed by economists to explain and predict human decision-making under risk and uncertainty. ${ }^{41}$ The fundamental assumption underlying utility theory is that the decision maker always chooses the alternative for which the expected value of the utility is maximized. Built into this assumption is a further supposition that a code of rationality is accepted and used by human decision-makers, thus making it possible to construct a mathematical representation that allows prediction of human behavior.

The basic approach of utility theory can become a foundational building block for a decision-making system intended for real-time autonomous control. Given a collection of seemingly viable alternative solutions, implementation risks determined for each alternative can be compared to find a minimum risk

\footnotetext{
${ }^{40}$ A. Wald, "Basic Ideas of a General Theory of Statistical Decision Rules," Proc. of the International Congress of Mathematicians, 1 (1950).

${ }^{41}$ D. Kahneman, A. Tversky, "Prospect Theory: An Analysis of Decision under Risk," Econometrica, v. 47-2 (March 1979).
} 
solution. Independent loss and gain (utility) functions as related to plant operating procedures or other decision strategies can be formulated and represented as nonlinear relationships.

\subsubsection{Markov Decision Processes}

Markov decision processes (MDPs) provide a mathematical framework for modeling decision-making in situations where outcomes are partly random and partly under the control of a decision maker. ${ }^{42}$ MDPs have been used successfully in a wide range of autonomous control problems ${ }^{43,44}$ and typically solve an optimization problem using dynamic programming (DP) for selecting the right decision. A partially observable MDP (POMDP) is a generalization of an MDP. ${ }^{45}$ A POMDP models a decision process in which it is assumed that the system dynamics are represented by an MDP, but not all states are observable. Instead, the measurements received by the model are incomplete and are usually noisy predictions. Therefore, the model must estimate a posterior distribution over a possible state space. ${ }^{46}$ POMDPs compute a value function, which is similar to a cost function in optimal control, over a belief space. A belief is a function of an entire probability distribution. An exact solution to a POMDP yields the optimal action for each possible belief over the state space, which maximizes the value function. However, this maximization procedure requires an iterative algorithm that is far from practical. For any reasonable number of states, sensors, and actuators, the complexity of the value function is prohibitive. Therefore, significant research has been conducted on the efficiency and optimality of solutions.

To incorporate the system dynamics into the decision-making process, the utility variables must be selected such that the projected physical behavior of the system can be factored in. One such approach uses utility attributes based on key process variables that have safety implications, such as trip set points. The utility values are then calculated based on the proximity of these critical variables to trip set points. ${ }^{47}$

\subsubsection{Discrete Event Systems}

Many engineering devices and systems and some natural systems demonstrate only discrete values or outcomes. Engineering systems are governed by operational rules designed by humans. For example, engineering systems are often considered to be either on or off, enabled or disabled, running or stopped. These types of systems are best described as discrete event systems (DESs). They are not easily analyzed, nor are they designed using conventional mathematics and engineering with time-driven methods represented by differential equations. Examples include transportation traffic systems, computer systems such as interrupts, communication systems, manufacturing processes, games, and queuing systems. Opening and closing of valves or commencing a pump start-up process are examples of discrete event processes in a nuclear power plant. These processes are typically tied to operating procedures, and their controls are handled by plant operators.

\footnotetext{
42 M. L. Puterman, Markov Decision Processes: Discrete Stochastic Programming, Wiley-Interscience, New Jersey (2005).

${ }^{43}$ S. Thrun, "Stanley: The Robot that Won the DARPA Grand Challenge," J. Field Robotics 23(9), 661-692 (2006).

${ }^{44}$ S. Brechtel, T. Gindele, and R. Dillmann, "Probabilistic Decision-Making under Uncertainty for Autonomous Driving using Continuous POMDPs," in 17th IEEE International Conference on Intelligent Transportation Systems, pp. 392-399 (2014).

${ }^{45}$ K. J. Åström, “Optimal Control of Markov Process with Incomplete State Information,” J. Math. Anal. App. 10, 174-205 (1965).

${ }^{46}$ S. Thrun, W. Burgard, and D. Fox, Probabilistic Robotics, The MIT Press, Cambridge, Massachusetts (2005).

${ }^{47}$ S. M. Cetiner et al., Development of a First-of-a-Kind Deterministic Decision-Making Tool for Supervisory Control System, ORNL/TM-2015/373, Oak Ridge National Laboratory (July 2015).
} 
DESs satisfy the properties that (1) state-space is a discrete set and (2) the state-transition mechanism is event-driven. Time in such systems is not the appropriate independent variable. Conventional differential equation approaches such as modern control theory do not apply to DESs. ${ }^{48}$ They are described as

\begin{abstract}
A class of dynamic systems characterized as synchronous or asynchronous occurrences of various discrete-valued events. Values are described by discrete values and transitions only occur at discrete points in time. Events are considered to occur instantaneously with some transition of one discrete value to another discrete value. These may be considered as time-driven or synchronous systems or event-driven or asynchronous systems.
\end{abstract}

DESs are typically modeled using an automata approach or a petri net approach. These approaches use a state-transition structure to describe the possible events in each state of the system. These two approaches differ in how they represent state information. An automaton is a device capable of representing a language according to well-defined rules and is commonly represented using a state-transition diagram with a defined set of states, initial states, events, and state-transition functions. Petri nets model state concurrency and synchronization in distributed systems using a combination of places, transitions, and directed $\operatorname{arcs}^{49}$.

\title{
5. ONGOING AND FUTURE WORK
}

The foregoing discussion points to several technical advances that are needed for autonomous operation and control in microreactors. These technical needs are potential targets for future research and development as part of technology maturation and include the following.

- Embedded sensors. Given the need to tightly couple sensors to the core and heat removal systems in microreactors, embedding some of these sensors in the core and support structures using advanced manufacturing processes is a possibility. However, identification of a minimal set of sensors that are essential for enabling autonomous operation is necessary and is planned as a future activity. This activity will likely identify two sets of measurement needs: one cutting across most microreactor concepts, one focusing on specific measurement sensor needs that are unique to a microreactor concept.

- Algorithms for condition assessment and decision-making. While several potential algorithms are available for these purposes, the challenges identified in this report will need to be addressed and the performance of the revised algorithms demonstrated on representative components and systems before incorporating them in autonomous control systems.

- Demonstration of autonomous operation. Such demonstration will require the identification of a near-term microreactor concept and focus on a predefined set of operational modes. Initial demonstrations will likely be simulation based (such as using TRANSFORM ${ }^{50}$ ) followed by hardware-in-the-loop demonstrations.

- Testbed for sensors, instrumentation, and control. As new sensors for microreactor monitoring are identified, a testbed where these sensors may be evaluated for accuracy, longevity, and reliability under representative conditions is needed. Ongoing efforts toward developing a

\footnotetext{
${ }^{48}$ C. G. Cassandras, S. Lafortune, Introduction to Discrete Event Systems (Second Edition), Springer (2008).

${ }^{49}$ Fortier, P. J., \& Michel, H. E. (2003). 9 - Petri Nets. In P. J. Fortier \& H. E. Michel (Eds.), Computer Systems Performance Evaluation and Prediction (pp. 279-303). Burlington: Digital Press.

${ }^{50}$ https://github.com/ORNL-Modelica/TRANSFORM-Library
} 
platform for high-temperature testing at US Department of Energy laboratories may be leveraged for this purpose. Other existing facilities for mocking-up reactor system operation and control may also be useful for this purpose. Such a testbed would also serve to demonstrate autonomous control technologies as part of a hardware-in-the-loop setup.

As noted above, the identification of a near-term microreactor concept is critical for successful development and demonstration of autonomous operation. Most concepts to date are either high-temperature gas-cooled concepts or use heat pipes for heat transfer from the core to the heat exchanger. It is likely that one of these concepts will be selected as an example for further development of the autonomous operation concept.

\section{CONCLUSIONS}

Most current microreactor concepts were conceived for mobile and remote siting and consist of concepts that operate at much higher temperatures than conventional light-water-cooled reactors. A range of factors drive the need for autonomous operation in these reactors, including the economics of operation, the need for rapid control response times when operating remotely, and the limited experience with operating many of these concepts over extended operating cycles with high capacity factors.

While autonomous control systems are gaining ground in a number of applications, their applicability to nuclear power plants has been limited. Several technical advances are needed before widespread use of autonomous control in microreactors, including sensor and instrumentation technologies for monitoring the reactor system and components, algorithms for inferring microreactor system state and for predictive decision-making, and actuators and control system hardware for unattended operation in harsh environments. Ongoing research and development is addressing several of these needs. 